\title{
Calculation of Zonal Winds Using Accelerometer and Rate Data from Mars Global Surveyor
}

\author{
Darren T. Baird* \\ Jet Propulsion Laboratory, California Institute of Technology, Pasadena, CA 91109-8099 \\ Robert Tolson $^{\dagger}$ \\ North Carolina State University, Hampton, VA 23666 \\ and \\ Stephen Bougher ${ }^{\ddagger}$ and Brian Steers ${ }^{\S}$ \\ University of Michigan, Ann Arbor, MI 48109-2143
}

\begin{abstract}
The Mars Global Surveyor spacecraft was initially placed into a high eccentricity, nearly polar orbit about Mars with a 45-hour period. To accomplish the science objectives of the mission, a 2-hour, circular orbit was required. Using a method known as aerobraking, numerous passes through the upper atmosphere slowed the spacecraft, thereby reducing the orbital period and eccentricity. To successfully perform aerobraking, the spacecraft was designed to be longitudinally, aerodynamically stable in pitch and yaw. Since the orbit is nearly polar, the yaw orientation of the spacecraft was sensitive to disturbances caused by the zonal components of wind (east-to-west or west-to-east) acting on the spacecraft at aerobraking altitudes. Zonal wind velocities were computed by equating the aerodynamic and inertia-related torques acting on the spacecraft. Comparisons of calculated zonal winds with those computed from the Mars Thermospheric Global Circulation Model are discussed.
\end{abstract}

\begin{tabular}{|c|c|c|c|c|c|c|}
\hline & \multicolumn{6}{|c|}{ Nomenclature } \\
\hline$A$ & $\begin{aligned}= & \text { Spacecraft } \\
& \text { reference area }\end{aligned}$ & $C_{z}$ & $\begin{aligned}= & Z \text { Z-direction force } \\
& \text { coefficient }\end{aligned}$ & $l, m, n$ & $=$ & $\begin{array}{l}\text { Direction cosine with } \\
\text { respect to } x, y, z \text { axes }\end{array}$ \\
\hline$B$ & $=$ Sensitivity matrix & $I$ & $\begin{aligned}= & \text { Moment of inertia matrix } \\
& \text { for single body }\end{aligned}$ & $N$ & $=$ & External torques (N-m) \\
\hline$C M$ & $=$ Center of mass & $I_{B / S}$ & $\begin{aligned}= & \text { Moment of inertia matrix } \\
& \text { for bus }+ \text { SAP assembly }\end{aligned}$ & $N_{g g}$ & $=$ & $\begin{array}{l}\text { Gravity gradient torque } \\
(\mathrm{N}-\mathrm{m})\end{array}$ \\
\hline$C_{m_{x}}$ & 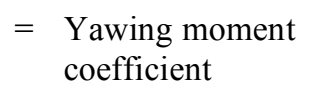 & $I_{S A M}$ & $\begin{aligned}= & \text { Moment of inertia matrix } \\
& \text { for SAM }\end{aligned}$ & $P x x x$ & $=$ & Periapsis for orbit $\mathrm{xxx}$ \\
\hline$C_{m_{y}}$ & $\begin{aligned}= & \text { Pitching moment } \\
& \text { coefficient }\end{aligned}$ & $J$ & $\begin{aligned}= & \text { Moment of inertia matrix } \\
& \text { for reaction wheels }\end{aligned}$ & $r_{s / c}$ & $=$ & $\begin{array}{l}\text { Areocentric spacecraft } \\
\text { position vector }\end{array}$ \\
\hline$C_{m_{z}}$ & $\begin{aligned} &= \text { Rolling moment } \\
& \text { coefficient }\end{aligned}$ & $L$ & $\begin{aligned} &= \text { Spacecraft reference } \\
& \text { length }\end{aligned}$ & $S A M$ & $=$ & $\begin{array}{l}\text { Solar array minus (-y } \\
\text { axis), broken panel }\end{array}$ \\
\hline$C_{x}$ & $\begin{aligned}= & \text { X-direction force } \\
& \text { coefficient }\end{aligned}$ & $L$ & $\begin{array}{l}=\text { Spacecraft angular } \\
\text { momentum }\end{array}$ & $S A P$ & $=$ & Solar array plus (+y axis) \\
\hline$C_{y}$ & $\begin{aligned}= & Y \text {-direction force } \\
& \text { coefficient }\end{aligned}$ & $L_{s}$ & $=$ Solar longitude & $\mathrm{S} / \mathrm{C}$ & $=$ & Spacecraft \\
\hline
\end{tabular}

\footnotetext{
${ }^{*}$ Member of Engineering Staff, Guidance, Navigation and Control Section, M/S 301-150, Lifetime Member AIAA.

${ }^{\dagger}$ Professor, Center for Planetary Atmospheric and Flight Sciences, 100 Exploration Way, Associate Fellow AIAA.

* Professor, Space Physics Research Laboratory, 2455 Hayward Avenue.

$\S^{\S}$ Student, Aerospace Engineering Department, 2455 Hayward Avenue, Student Member AIAA.
} 


\section{Introduction}

A fter a 10-month cruise from Earth, Mars Global Surveyor (MGS) was placed into a highly elliptical, nearly polar orbit about Mars. MGS was the first interplanetary probe where mission success was dependent on the safe, timely, and effective used of aerobraking. The goal of aerobraking was to reduce the period of the nearly polar orbit from 45 hours to 2 hours without relying on propulsive burns. Aerobraking was broken into two phases and used over 1200 controlled passes through the upper atmosphere to gradually slow the spacecraft, thereby reducing the orbital period and eccentricity. ${ }^{1}$

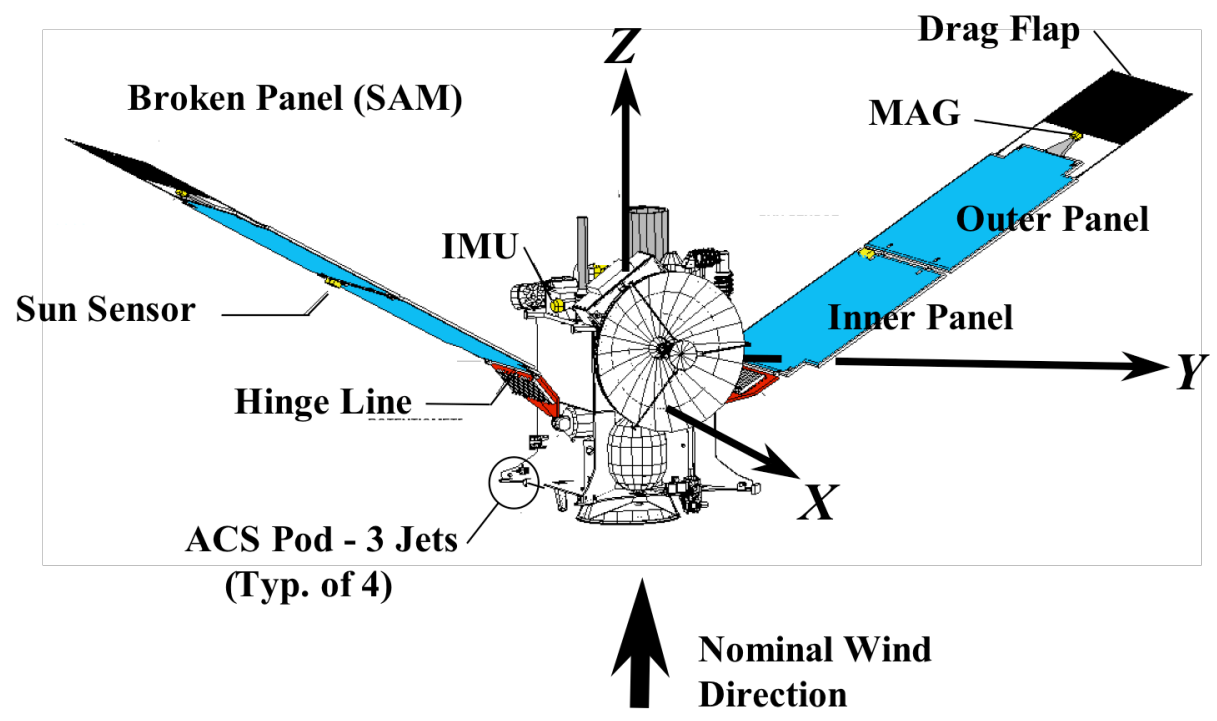

During each aerobraking pass the solar arrays were swept aft approximately $30^{\circ}$, which shifted the center of pressure aft of the center of mass of the spacecraft. This configuration, shown in Fig. 1, provided longitudal, aerodynamic stability in pitch and yaw throughout each aerobraking pass. Figure 1 also shows that the normal flow is along the z-axis, but preflight attitude control

Figure 1. MGS Aerobraking Schematic. simulations indicated that during the aerobraking passes, the relative winds

could deviate from the z-axis by as much as $15^{\circ}$. Since the orbit is nearly polar, the yaw orientation of the spacecraft was sensitive to disturbances caused by zonal components of wind (east-to-west or west-to-east) acting on the spacecraft at aerobraking altitudes, as shown in Fig. 2.

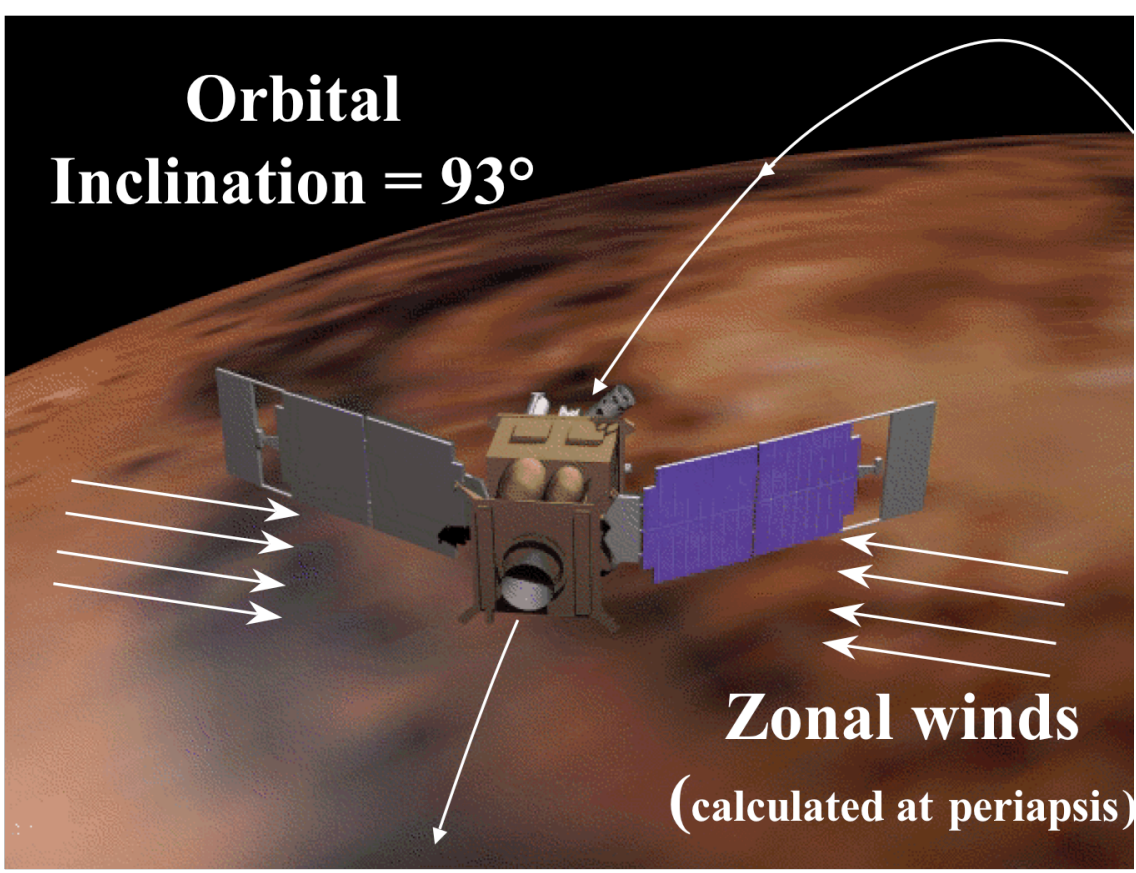

As will be shown, accelerometers, rate gyros, and reaction wheels onboard MGS can serve as instruments to calculate these winds. Accelerometer data are first used to calculate the density profiles throughout each atmospheric pass. The reaction wheel data are used to calculate the effects of onboard momentum sources. Euler's equations of motion are then used to relate the aerodynamic and gravity gradient torques to the attitude motion of the spacecraft. By solving these equations, it is possible to determine what torques the spacecraft experiences due to local winds and, therefore, calculate the components of

Figure 2. Zonal wind components acting on MGS. wind acting on the vehicle. 


\section{Euler's Equations of Motion}

To determine zonal winds, accelerometer data are first used to calculate the density of the atmosphere for the duration of each aerobraking pass using Eq. $1^{2,3}$, where $\mathrm{m}$ is spacecraft mass, $\mathrm{v}$ is spacecraft velocity, $\mathrm{C}_{\mathrm{d}}$ is drag coefficient, and A is the spacecraft cross-sectional area in the aerobraking configuration.

$$
\rho=\left[2 m / v^{2} C_{d} A\right] \dot{v}
$$

The reaction wheel data are used to calculate the effects of onboard momentum sources. Euler's equations of motion are then used to relate the aerodynamic and gravity gradient torques to the attitude motion of the spacecraft. By solving these equations, it is possible to determine what torques the spacecraft experiences due to local winds and, therefore, calculate the components of wind acting on the vehicle.

For a rigid body, attitude motion is represented by Euler's equations, as shown in Eq. 2. The total angular momentum of the spacecraft is represented by $\bar{L}$, with contributions from the Solar Array Minus (SAM), bus/Solar Array Plus (bus/SAP) assembly, and the reaction wheels, while $\bar{N}$ represents the externally generated torques caused by gravity gradient, aerodynamics, and thruster fires.

$$
\frac{d \bar{L}}{d t}=\bar{N}
$$

Since Euler's equations of motion relate external torques to the time rate of change of angular momentum of the spacecraft, the torques acted upon the spacecraft by zonal winds can be calculated if all other external torques and the time rate of change of angular momentum can be determined. These external torques applied to the spacecraft include thruster firings, gravity gradient, and aerodynamics, while desaturation of the reaction wheels and the deflection of the Solar Array Minus (SAM) generate internal torques. These torques are calculated using spacecraft telemetry from the corresponding aerobraking pass.

\section{A. Equations of Motion for Two Rigid Bodies}

Because of the broken solar panel, MGS is modeled as 2 rigid bodies connected by a non-linear, torsional spring. The two bodies are the SAM and bus/Solar Array Plus (bus/SAP) combination. Euler's equations can be written independently for each body with respect to an inertial frame as shown in Eq. 3.

$$
\frac{d \bar{L}}{d t}=\bar{N}=\frac{d(I \bar{\omega})}{d t}=I \dot{\omega}+\dot{I} \omega
$$

Calculating the time rate of change of the moments of inertia (I) is difficult. However, an analysis was performed assuming a SAM deflection of $5^{\circ}$, which corresponds to a maximum angular velocity of $0.0819 \mathrm{rad} / \mathrm{sec}$. This analysis demonstrated that the last term of Eq. 3 is negligible for the $y$ and $\mathrm{z}$ axes. More importantly, this term is zero for the $\mathrm{x}$-axis, and since the zonal winds cause torques primarily about the $\mathrm{x}$ axis, the last term of Eq. 3 was disregarded. Rotating, body-fixed systems were chosen about the center of mass of the SAM and bus/SAP combination so that the moments of inertia of each body remain constant. When adding the angular momentum terms, a common basis must be referenced, which was the spacecraft center of mass with no SAM deflection. Therefore, Euler's equation for the two-body system is represented by Eq. 4.

$$
\begin{aligned}
& I_{B / S} \dot{\varpi}_{B / S}+\varpi_{B / S} \times I_{B / S}{ }_{B / S}+J \dot{\Omega}+\varpi_{B / S} \times J \Omega+I_{S A M} \dot{\varpi}_{S A M}+ \\
& { }_{S A M} \times{ }_{S A M}{ }_{S A M}-\bar{N}_{g g}=0.5 \rho \bar{V}_{S / C}^{2} A L C_{m_{x}}
\end{aligned}
$$


The known terms (i.e. - those obtained from attitude control system data) that do not depend on zonal winds are moved to the left-hand side of Eq. 4 to yield the equation to be evaluated. The known terms are evaluated using the two body-fixed coordinate systems, one centered at the center of mass of the bus/SAP assembly and the other centered at the SAM center of mass. $\mathrm{C}_{\mathrm{m}_{\mathrm{X}}}$ is the only term that significantly depends on zonal winds, and Fig. 3 shows $\mathrm{C}_{\mathrm{x}}$ as a function of yaw angle for panel deflections of $0^{\circ}, 10^{\circ}, 17^{\circ}$, and $20^{\circ}$ in free molecular flow

Figure 3 shows that the panel deflection causes a shift in $\mathrm{C}_{\mathrm{m}_{\mathrm{X}}}$ such that a $10^{\circ}$ panel deflection produces about $5^{\circ}$ of displacement in the equilibrium yaw angle. Also, $\mathrm{C}_{\mathrm{m}_{\mathrm{X}}}$ varies linearly with the $y$-component of wind

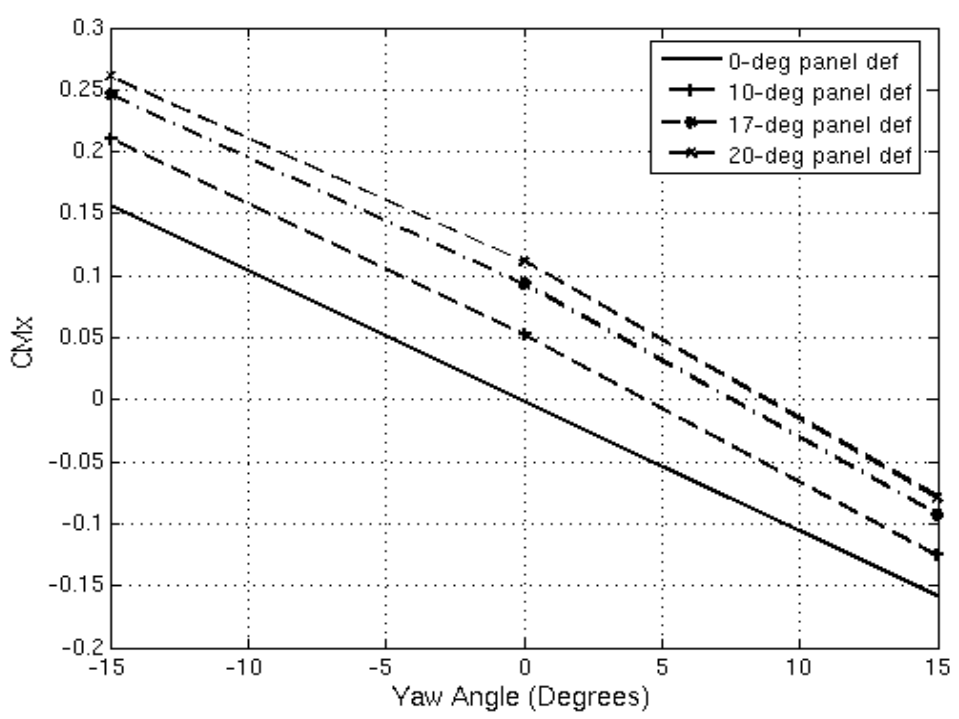

Figure 3. $C_{m_{x}}$ as function of relative wind for different panel deflections. for a given dynamic pressure. Zonal winds cause a change in yaw angle, and $\mathrm{C}_{\mathrm{m}_{\mathrm{X}}}$ must be calculated to determine zonal winds.

The attitude control system (ACS) of MGS consists of rate gyros, reaction wheels, thrusters, and sun sensors. The angular velocity of the bus, $\omega_{\mathrm{B} / \mathrm{S}}$, was measured by the bus-fixed gyros, and these data were recorded at 1 second intervals throughout each aerobraking pass. Using a double-sided difference, the time derivatives of $\omega_{\mathrm{B} / \mathrm{S}}$ were calculated. The angular velocity of the spacecraft near Periapsis-52 (P52) is shown in Fig. 4.

The low frequency variations in Fig. 4 are due to aerodynamic moments and have a characteristic time of approximately 50 seconds. Also, Fig. 4 shows a 6-second oscillation $(0.15 \mathrm{~Hz})$ caused by the SAM deflection

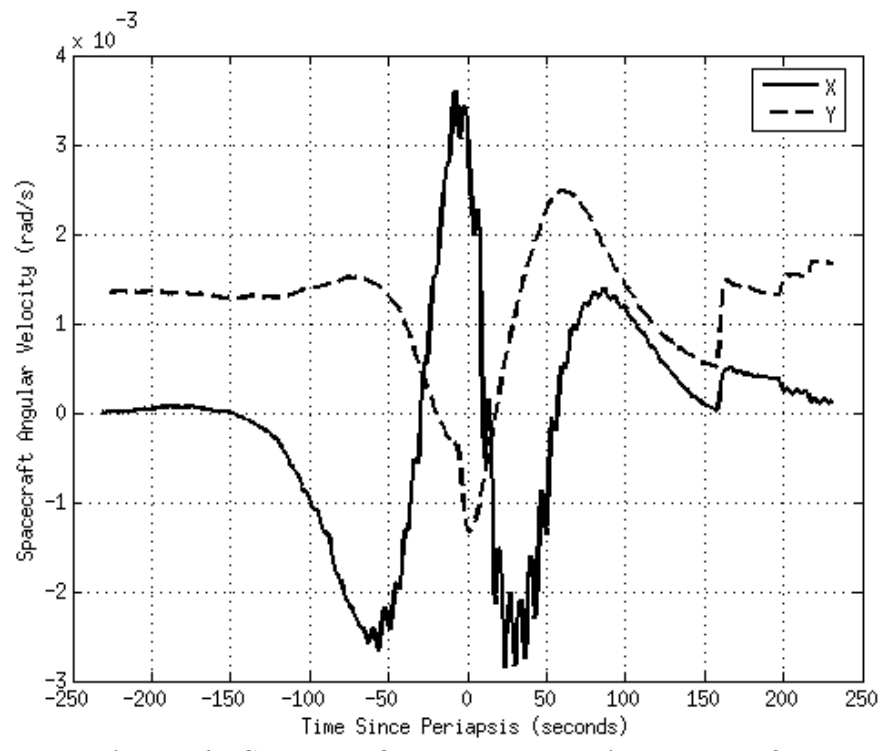

Figure 4. Spacecraft angular velocity near P52. throughout the aerobraking pass. As the SAM oscillates, the bus must counter balance the motion to preserve angular momentum if external torques are ignored. This 6-second oscillation is not of interest in the determination of zonal winds, and all data were filtered using a low pass, 2-direction filter.

Panel position data were calculated at 1-second intervals throughout each aerobraking pass. ${ }^{4}$ Angular velocities of the SAM, $\omega_{\text {SAM }}$, were calculated using a double-sided difference of the filtered panel position, and angular accelerations were similarly calculated using a doublesided difference of the angular velocities.

Referring to Fig. 4, about 10 seconds before periapsis there is a change in angular velocity, shown by a sudden change in the slope in the y-axis angular momentum. This angular acceleration is due to a reaction wheel desaturation, which usually occurs near periapsis, Reaction wheels were not used for attitude control during aerobraking 
because they cannot produce enough torque to overcome the moments produced by the aerodynamic forces, so their speeds are held nearly constant. However, to minimize attitude control fuel usage, reaction wheel biasing events were performed near periapsis, where aerodynamic torques easily balanced the reaction wheel torques due to desaturation. For purposes of this method, a reaction wheel biasing event refers to the change in velocity from one nominal speed before periapsis to another nominal speed after periapsis. During each reaction wheel biasing event, the reaction wheel speeds are changed at a nearly constant acceleration.

Knowing that the three reaction wheels start desaturating at the same time, the maximum difference between the mean velocities of the reaction wheels before and after the biasing event is found and a best-fit line is drawn through the points where the reaction wheel velocity is changing. The time where this line intercepts the mean velocity before the biasing event began is taken to be the bias event start time. Figure 5 shows the original data, the linear fit

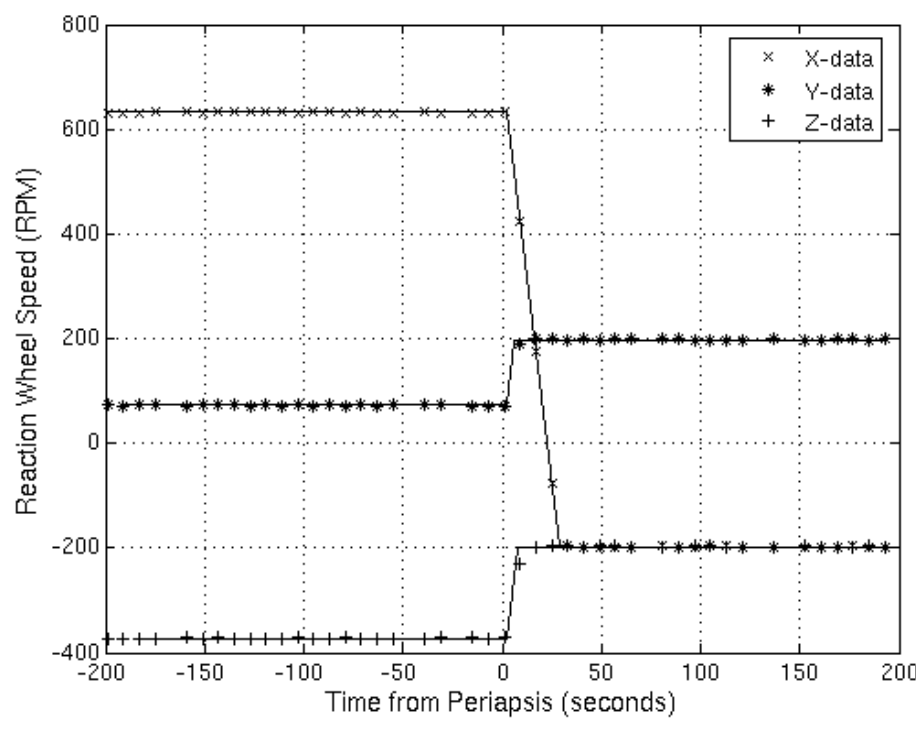

Figure 5. Reaction wheel data for P52. to the data, and the lines of mean reaction wheel speed before and after the biasing event, as represented by data from P52.

The mean reaction wheel speeds before the biasing event are used as the reaction wheel speeds for all times up to the biasing event start time, and the mean reaction wheels speeds after the biasing event are considered as the reaction wheel speeds for all times after the biasing event.

\section{B. Zonal Wind Terms}

As MGS passed through the atmosphere in a nearly polar orbit, three separate wind components act on the spacecraft. Vertical winds are generally on the order of $10 \mathrm{~m} / \mathrm{s}$ and are not expected to greatly affect the spacecraft attitude motion. Meridional winds, which are in the north/south direction, also do not greatly affect the spacecraft heading because MGS is in a nearly polar orbit, and these winds contribute no more than a 100 $\mathrm{m} / \mathrm{s}$ velocity change along the orbital velocity vector and do not produce attitude changes. However, the zonal winds, which travel in the east/west direction (cross winds with respect to the spacecraft), can significantly change the spacecraft heading, as illustrated in Fig. 2. For example, at P50, the spacecraft velocity was $4,760 \mathrm{~m} / \mathrm{s}$ at $38.4^{\circ}$ north latitude and at an altitude of $123.5 \mathrm{~km}$. Under those conditions, the inertial zonal winds at periapsis were calculated by taking the cross product of the rotation rate of the planet and the spacecraft position vector. The wind velocity is $171 \mathrm{~m} / \mathrm{s}$, which causes the heading to change by approximately $2^{\circ}$. It is normally assumed that the atmosphere is rigidly rotating about the polar axis at a constant angular velocity, $\bar{\lambda}$, throughout the entire aerobraking pass. Under this condition, an observer fixed on the planet would not detect any winds. By adding or subtracting the rigid rotation component from the inertial zonal winds, Eq. (5) can be used to calculate the wind velocity with respect to the spacecraft.

$$
\vec{V}_{\text {Flow }}=\vec{V}_{S / C}+\operatorname{Super}\left(\vec{\lambda} \times \vec{r}_{s / c}\right)
$$

The gravity gradient torques were calculated as shown in Eq. $6 .{ }^{5}$ The values 1 , $\mathrm{m}$, and $\mathrm{n}$ are the direction cosines in the body system of the vector extending from the spacecraft to the center of Mars, and $r$ is the distance from the spacecraft center of mass to the center of Mars. 


$$
\begin{aligned}
& N_{X_{g z}}=\left(3 \mu_{\text {Mars }} / r^{3}\right)\left[m n\left(I_{z z}-I_{y y}\right)+n l I_{x y}-l m I_{x z}+\left(n^{2}-m^{2}\right) I_{y z}\right] \\
& N_{Y_{g g}}=\left(3 \mu_{\text {Mars }} / r^{3}\right)\left[n l\left(I_{x x}-I_{z z}\right)+l m I_{y z}-m n I_{x y}+\left(l^{2}-n^{2}\right) I_{x z}\right] \\
& N_{Z_{g g}}=\left(3 \mu_{\text {Mars }} / r^{3}\right)\left[n l\left(I_{y y}-I_{x x}\right)+m n I_{x z}-n l I_{y z}+\left(m^{2}-l^{2}\right) I_{x y}\right]
\end{aligned}
$$

Gravity gradient torques are small compared to the other torques, but for completeness they were retained to eliminate a source of error.

\section{Aerodynamic Torques}

To evaluate the right-hand side of Eq. 4, the velocity of the wind relative to the spacecraft must be known. The velocity of the wind relative to the spacecraft is the parameterized rotation of the atmosphere using a parameter called super rotation, shown in Eq. 5. A super rotation rate (Super) of 1 means that the atmosphere is rotating at the same rate as the planet. Super greater than 0 but less than 1 means that westerly winds are blowing, but they are slower than the rotation rate of the planet. Super greater than 1 means westerly winds are being experienced that are faster than the rotation rate of the planet. If Super is less than zero, the winds are easterly. Super was initially assumed to be one. The spacecraft velocity relative to the winds was transformed into a body-fixed coordinate system by pre-multiplying the spacecraft position vector by a rotation matrix. This transformation yielded the velocity components of the wind relative to the spacecraft, called $\bar{V}$.

To solve the aerodynamic torque equations, moment and force coefficients must be calculated. Multi-degree interpolation over a range of atmosphere densities from free molecular flow to deep in the transition region, a range of wind components, and a range of panel deflections was used to find values for $\mathrm{C}_{\mathrm{m}_{\mathrm{X}}}, \mathrm{C}_{\mathrm{m}_{\mathrm{y}}}, \mathrm{C}_{\mathrm{x}}$ and $\mathrm{C}_{\mathrm{y}}$ that corresponded to the known density and panel deflection at each point during the pass. The coefficients in the aero tables, each of which corresponded to a different panel deflection of $0^{\circ}, 10^{\circ}$, and $20^{\circ}{ }^{6}$, were referred to the assumed spacecraft center of mass.

The original aerodynamic coefficients were not calculated about the actual spacecraft center of mass. Therefore, the center of mass of the spacecraft had to be shifted to the location were these coefficients are valid. Using the initial spacecraft center of mass location as the "origin," the shifts in the $\mathrm{x}, \mathrm{y}$, and $\mathrm{z}$ directions to the location where the aerodynamic coefficients were calculated are represented by $\Delta \mathrm{x}, \Delta \mathrm{y}$, and $\Delta \mathrm{z}$, which are all constants. However, the spacecraft center of mass changed as the SAM deflected, and these shifts in the $y$ and directions are represented by $\delta \mathrm{y}$ and $\delta$ z. The aerodynamic torque equations, therefore, take the form show in Eq. 7, where $\mathrm{C}_{\mathrm{x}}$ and $\mathrm{C}_{\mathrm{y}}$ depend on the super rotation parameter.

$$
\begin{aligned}
& N_{x}=0.5 \rho V^{2} A L \frac{\left(C_{m_{X}}+\left[(\Delta y+\delta y) C_{z}-(\Delta z+\delta z) C_{y}\right]\right)}{L} \\
& N_{y}=0.5 \rho V^{2} A L \frac{\left(C_{m_{y}}+\left[(\Delta z+\delta z) C_{x}-\Delta x C_{z}\right]\right)}{L}
\end{aligned}
$$

\section{Least Squares Solution}

The least squares method was used in an iterative manner to solve for zonal winds over a batch or orbits. The purpose of this differential correction was to minimize the difference between the inertia-related and aerodynamic torques in Eq. 4 over arbitrary batches of orbits by varying the spacecraft center of mass location and the super rotation rate. For a single orbit, it is impossible to solve for both zonal winds and the center of mass because of the high correlation between $\Delta \mathrm{z}$ and the super rotation parameter.

To find the least squares solution, the left-hand side (LHS) and right-hand side (RHS) of Eq. 4 are balanced. That is, LHS $=$ RHS $+\varepsilon$, where the parameters are chosen to minimize the length of the residual vector, $\varepsilon$. To perform the least squares minimization, the sensitivity matrix is calculated by finite difference partials. The sensitivity matrix consists of four separate partials, which are found by taking the partial derivatives of the known terms with respect to the three center of mass locations $(\Delta \mathrm{x}, \Delta \mathrm{y}, \Delta \mathrm{z})$ and a super rotation parameter for each orbit. 
Upon completion of the iteration process, the super rotations that minimized the error of the least squares equations for every orbit were determined. The center of the mass of the spacecraft was held constant for the batch, and the super rotation values yielded the zonal winds.

A flow chart is included in the appendix that demonstrates the processes used to calculate zonal wind velocity.

\section{Spacecraft Geometry}

The determination of zonal winds is highly dependent on an accurate knowledge of the spacecraft geometry. The common basis used to solve Eq. (5) was the spacecraft center of mass, but components are often referenced with respect to the nozzle exit plane. Figure 6 shows the dimensions of each major component with respect to the center of mass of the spacecraft.

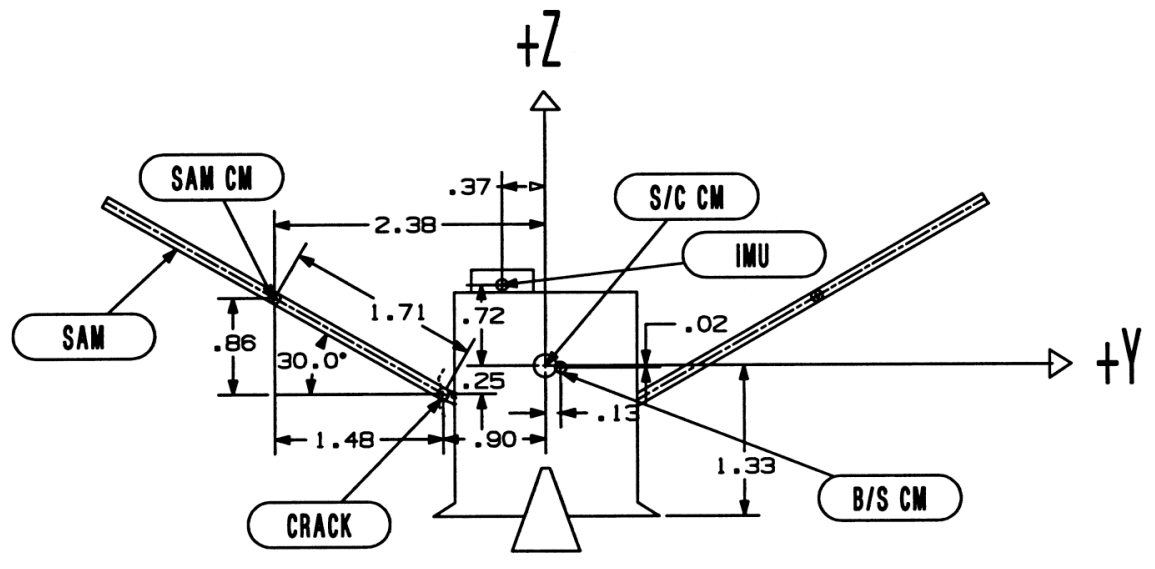

Figure 6. MGS Geometry (dimensions in meters).
The average mass of the entire spacecraft is 757 $\mathrm{kg}$, with the SAM weighing $39.34 \mathrm{~kg}$ and the bus/SAP combination weighting an average of $717.66 \mathrm{~kg}$.

\section{Effects of Solar Array Minus (SAM) Deflection}

The SAM deflected about the $\mathrm{x}$-axis during aerobraking passes because a solar array deployment damper failed during the cruise phase of the mission, thereby causing

the partial structural failure of the SAM. ${ }^{7}$ This panel anomaly is important in the determination of zonal winds because the panels may have different accommodation coefficients, and there exists a panel bias which varies during the course of aerobraking.

\section{A. Effects of Different Accommodation Coefficients}

Gas-surface accommodation coefficients represent the degree to which incident molecules achieve equilibrium with the surface. Incomplete accommodation (accommodation less than 1) reduces the momentum and energy of particles transferred to the surface. As part of the aerobraking operations, the SAM was rotated $180^{\circ}$ to attenuate the stresses acting on the panel. Therefore, the forward facing surface of the SAM is glass-covered solar cells, while the forward facing surface of Solar Array Plus (SAP) is a composite face sheet. Glass has a lower accommodation coefficient than a composite face sheet, and differences in accommodation coefficients up to 0.2 may be possible, based on limited observations of the Magellan spacecraft. ${ }^{6}$ A lower accommodation coefficient on the SAM means that there is a greater degree of specular reflection, and forces are higher in the stream direction, which produces a moment that rotates the spacecraft toward SAM. This effect causes the spacecraft to trim at yaw angles other than zero, which was assumed in the calculation of the zonal winds. Even though the trimmed yaw angle is less than $1^{\circ}$ for typical periapsis densities, the spacecraft was rolled about the y-axis $180^{\circ}$ near P138. Subsequently, MGS assumed a different equilibrium yaw angle with respect to the planet, while the zonal winds were still the same. Since zonal wind velocity is a function of yaw angle, discontinuities in the results exist near the orbit where the roll was executed. Since there is no way of accurately determining the yaw angle bias induced by the differential accommodation coefficients during each pass, it is considered an acceptable, albeit uncorrectable, source of error.

\section{B. Effect of Panel Bias}

The zonal wind velocities are highly dependent on an accurate knowledge of the spacecraft center of mass location. As the SAM deflects, the center of mass of the spacecraft is shifted in both the $\mathrm{y}$ and $\mathrm{z}$ direction as shown in Eq. 8. 


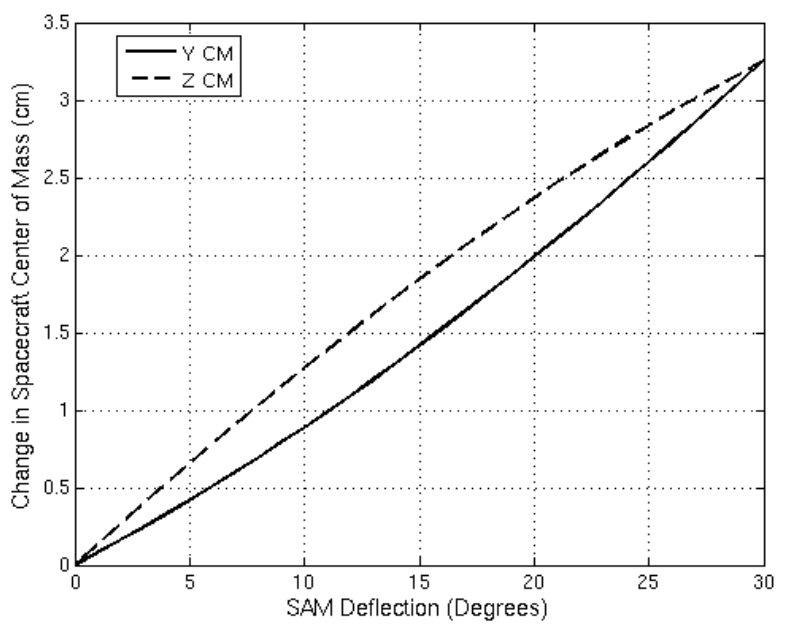

Figure 7. Center of mass shift due to panel deflection.

$\Delta C M_{S / C}=\frac{\left(M_{S A M} \Delta C M_{S A M}\right)}{M_{S / C}}$

Figure 7 shows the change of spacecraft center of mass in the $\mathrm{y}$ and $\mathrm{z}$ directions as a function of SAM deflection.

A change in spacecraft center of mass due to SAM deflection results in a corresponding change in $\mathrm{C}_{\mathrm{m}_{\mathrm{X}}}$, which must be accurately calculated to solve for zonal wind velocity. $\mathrm{C}_{\mathrm{m}_{\mathrm{X}}}$ varies as SAM deflection changes according to Eq. 9 and 10,

The changes in $\mathrm{C}_{\mathrm{m}_{\mathrm{X}}}$ due to SAM deflection are shown in Fig. 3. $\mathrm{C}_{\mathrm{m}_{\mathrm{X}}}$ has a negative slope, indicating positive stability of the spacecraft. It also has an equilibrium position at a yaw angle of $0^{\circ}$ with no SAM deflection. With a SAM deflection of $10^{\circ}$, a yaw angle of $5^{\circ}$ would be necessary for the spacecraft to maintain a state of equilibrium, resulting in $\mathrm{C}_{\mathrm{m}_{\mathrm{X}}}$ shifting from 0 to -0.01 . Therefore, the effects of panel position on the spacecraft center of mass are not negligible when calculating zonal wind velocity.

The SAM experienced a bias during both mission phases. The effects of panel deflection about $\mathrm{x}$ were taken into account during calculation of zonal wind velocity by using a panel deflection model, assuming the SAM is joined to the bus with a non-linear torsional spring. However, a drifting bias occurred before and after periapsis in both phases, with the largest effect seen in phase 1.

$$
\begin{aligned}
C_{m_{x}} & =C_{m_{x 0}}+\frac{\left(\delta y_{S A M} \cdot C_{z}-\delta z_{S A M} \cdot C_{y}\right)}{L} \\
C_{m_{y}} & =C_{m_{y 0}}+\frac{\left(\delta z_{S A M} \cdot C_{x}-\delta x_{S A M} \cdot C_{z}\right)}{L}
\end{aligned}
$$

Since zonal wind velocity is highly dependent on the spacecraft center of mass location, which is dependent on the SAM position, this bias cannot be ignored. The bias reached its maximum value of $2^{\circ}$ on Orbit-152. A large discontinuity occurred near Orbit-80, where the bias actually changed direction. The cause of this sudden change is not known, but one theory attributed this change to the $0.16 \mathrm{~Hz}$ mode being strongly excited on some orbits, which caused the cracked face sheets to suddenly slip past each other. The bias was taken into account by fitting a curve through the inbound and outbound measured panel deflection data. The average cubic polynomial between the inbound and outbound was calculated, and this model was assumed to be the panel bias near periapsis, the region where zonal wind velocities are calculated. A bias discontinuity also exists between the end of phase 1 and the beginning of phase 2 , and it is postulated that this change was induced by the numerous attitude changes that occurred during the science phasing orbits.

Figure $8 \mathrm{a}$ shows the inbound, outbound, and average panel bias for phase 1, while Fig. $8 \mathrm{~b}$ shows the inbound, outbound, and average panel bias for phase 2 up through P992. Subsequent orbits are not used to calculate zonal winds due to the low orbital eccentricity, which results in a large latitudinal change during the aerobraking passes. Zonal winds are assumed to be constant throughout the entire pass and are meaningless over a wide array of latitudes. 


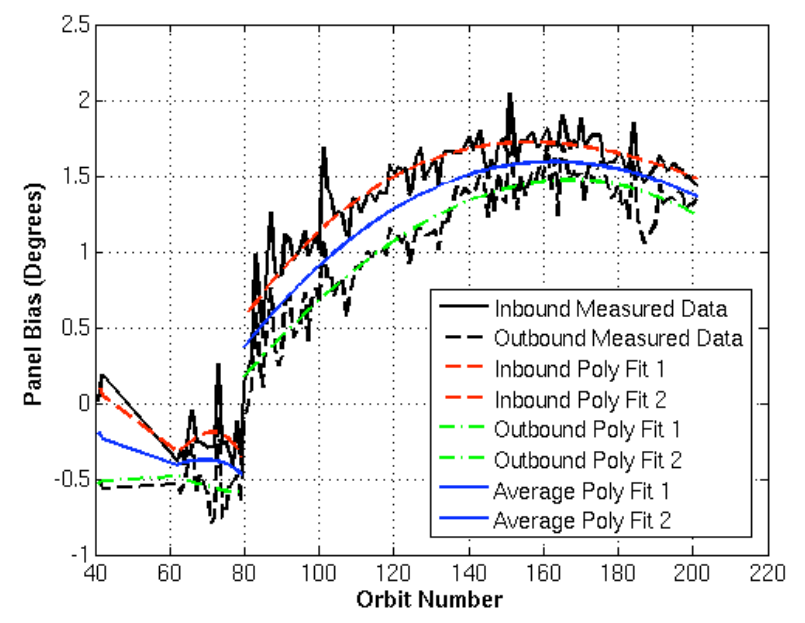

a) Phase 1

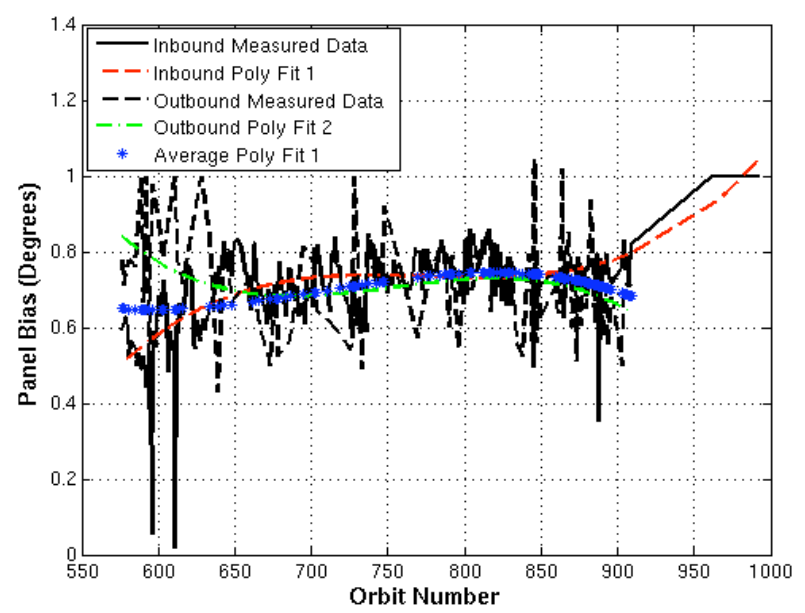

b) Phase 2

Figure 8. Panel Bias and Polynomial Fits.

\section{Results}

For each aerobraking pass, the X-torques and Y-torques are calculated from the left-hand and right-hand sides of Eq. 4 while taking into account the SAM bias. Confidence in the model is gained when plots of the external and inertia-related torques compare well. For most orbits, both the x-torques and y-torques have good correlation between the left and right-hand sides of Eq. 2, but if the correlation coefficient falls below 0.9 , the winds from that orbit are not calculated.

Generally, the aerodynamic torques are less than the inertia-related torques, but the deviations between the known torques and aerodynamic torques are small enough to make the potential error in zonal wind calculations acceptable $(\sim 5 \mathrm{~m} / \mathrm{s} 1 \sigma)$.

The least squares process is run in batches of approximately ten orbits each, and the super rotation rate is calculated for each orbit while the spacecraft center of mass is held constant for all orbits in the batch. The zonal wind velocity at periapsis is calculated using Eq. 5 .

\section{A. Phase 1 Winds}

Figure 9 shows that the phase 1 zonal wind magnitude at periapsis reached a high value of approximately $\sim 300$ $\mathrm{m} / \mathrm{s}$ during P53. This high velocity was reached at the same time as a large dust storm erupted in the Noachis Terra region of Mars in November 1997. P53 occurred during the southern spring season, a season that usually marks the onset of maximum dust storm activity. In general, the phase 1 zonal winds are observed to be westerly (west to east flow) at the periapsis altitude of $\sim 120-130 \mathrm{~km}$. 


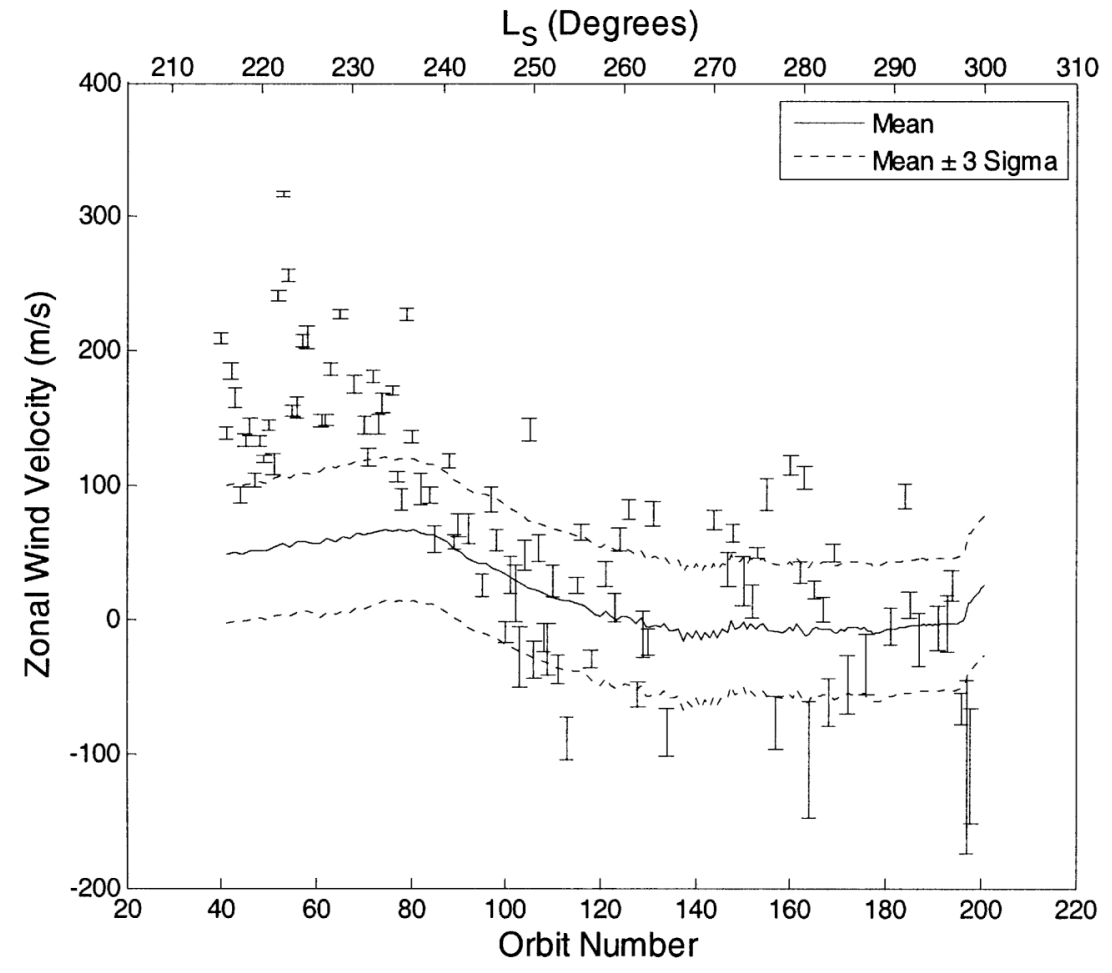

Figure 9. Computed phase 1 zonal winds with 3- $\sigma$ error bars superimposed on mean and $\pm 3-\sigma$ winds computed from MARSGRAM 2005 .
This wind speed experienced a downward trend as latitude varied in a nearly linear fashion from $37^{\circ}-61^{\circ} \mathrm{N}$ over P40-P201. Immediately before the onset (prior to P49) and after the Noachis storm decay (beyond P90), zonal wind magnitudes are $\sim 75-100$ $\pm 3 \mathrm{~m} / \mathrm{s} \quad(1 \sigma$, westerly). Maximum dust storm winds (near P53) are approximately $\sim 200 \mathrm{~m} / \mathrm{s}$ stronger. Over the higher latitude regions (beyond P100), the zonal wind magnitudes decreased significantly, and in some instances, are noticed to be easterly (east to west flow).

When MGS was at periapsis $\mathrm{P} 40$, the season was southern spring (solar longitude $\left.\left(L_{S}\right)=215\right)$, but by orbit 201, the southern summer season $\left(\mathrm{L}_{\mathrm{S}} \sim 300\right)$ had arrived. The Northern hemisphere $\left(37^{\circ}-61^{\circ} \mathrm{N}\right)$ wind velocities tend to decrease from southern spring to summer (northern fall to winter). The amount of wind variation due to latitudinal effects and season effects is discussed in subsequent sections of this report.

Superimposed on this plot of observed zonal wind magnitudes are simulated zonal winds obtained from the MARSGRAM-2005 (MG2005) empirical model. ${ }^{8}$ These calculated winds are provided for the periapsis location (latitude, longitude, solar local time, altitude), seasonal conditions ( $\mathrm{L}_{\mathrm{S}}$, dust opacities), and solar fluxes encountered during MGS phase 1 aerobraking for orbits P40-P201. An average integrated visible dust opacity of $\sim 1.0$ is assumed throughout these phase 1 orbits, in accord with observed opacities outside the Noachis dust storm period ( P49P90). Simulated mean and $\pm 3-\sigma$ curves for zonal wind magnitudes are given. It is clear that MG2005 simulations capture the basic features of the zonal wind trends with latitude and season during this interval, except during the Noachis dust storm onset and decay period. During this time, observed zonal winds are much stronger (up to $\sim 300$ $\mathrm{m} / \mathrm{s}$ ). The MG2005 model was constructed from coupled model simulations of the NASA Ames Mars Global Circulation Model (MGCM) and the NCAR/Michigan Mars Thermospheric General Circulation Model (MTGCM) for specific Mars seasonal, dust, and solar conditions. ${ }^{8,9}$ No attempt was made to provide simulated evolving dust storm outputs to the MG2005 framework. The derived zonal winds presented in Fig. 9 provide a strong constraint for such a comprehensive evolving dust storm simulation using the coupled MGCM-MTGCM codes. 
Figure 10 shows the same derived zonal winds, now superimposed with MG2005 winds calculated using a simplified dust storm evolution scheme. In this instance, dust opacities are assigned to vary from 0.4 to 1.0 during the Noachis dust storm onset, and to decay thereafter. The effect on the simulated zonal winds is modest, resulting in slightly larger wind speeds during the storm itself $(+10-15 \mathrm{~m} / \mathrm{s})$, and a more gradual decline of zonal winds speeds over P90 to P140. In both cases (Fig. 9 and Fig. 10), simulated dust storm period ( $\mathrm{L}_{\mathrm{S}} \sim 220$ to 240 ) zonal winds are

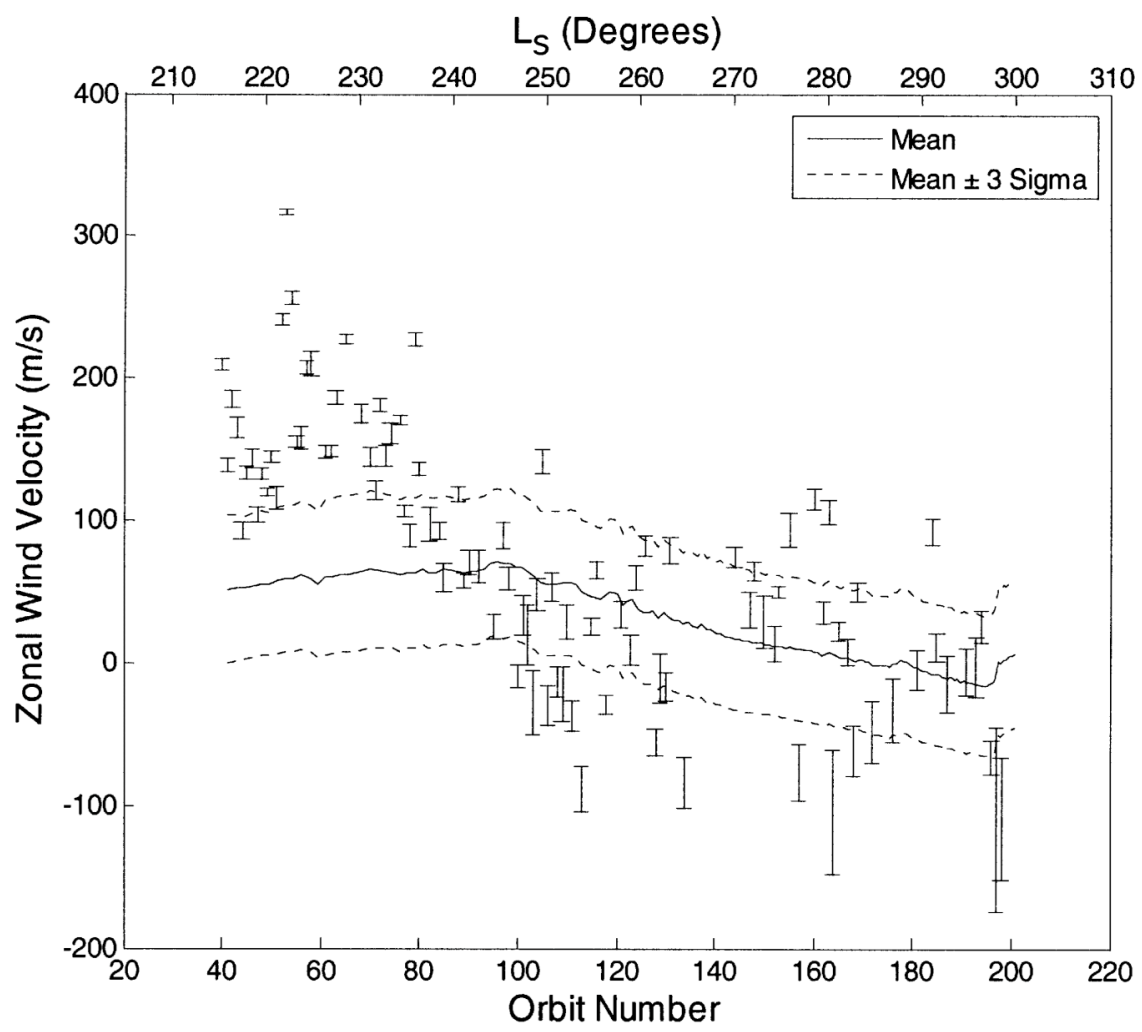

Figure 10. Computed phase 1 zonal winds with 3- $\sigma$ error bars superimposed on mean and $\pm 3-\sigma$ winds computed from MARSGRAM 2005 with dust storm evolution scheme. much weaker than observed. Again, the case is made for conducting a comprehensive evolving dust storm simulation using the coupled MGCM-MTGCM codes. The radiative, dynamical, and dust transport feedbacks present in the Mars atmosphere during the Noachis storm, giving rise to these zonal wind variations, can then be investigated.

\section{B. Phase 2 Winds}

The phase 2 zonal wind velocities are calculated using the same methods as in phase 1. Again, for those orbits, when the $\mathrm{x}$-torques and $\mathrm{y}$ torques have a correlation coefficient that falls below 0.9 , the winds from those orbits are not calculated. For all these orbits, SAM bias is taken into account. Furthermore, it is recognized that phase 2 orbits occurred during the MGS aerobraking period when orbit periods were less than 12.5 hours, and

the spacecraft was in the atmosphere (below $200 \mathrm{~km}$ ) for more than $500 \mathrm{sec}$ on each aeropass. This long residence time in the atmosphere required many spacecraft thruster firings (more than phase 1), for which numerous individual corrections are needed before useful wind speeds can be derived. These corrections are not applied in this exercise.

Figure 11 illustrates phase 2 zonal wind magnitudes spanning $\mathrm{L}_{\mathrm{S}}=35^{\circ}-80^{\circ}(\mathrm{P} 550-\mathrm{P} 992)$. This corresponds to a period from early $\left(\mathrm{L}_{\mathrm{S}} \sim 35\right)$ to late $\left(\mathrm{L}_{\mathrm{S}} \sim 80\right)$ northern spring, as the spacecraft periapsis moved from $60^{\circ} \mathrm{N}$ to the South pole. Wind magnitudes show a high degree of orbit-to-orbit variability in this plot, with zonal winds generally decreasing during early northern spring (southern fall) and then increasing during late northern spring (southern fall). Wind speeds range from approximately $+150 \mathrm{~m} / \mathrm{s}$ (westerly) to $-125 \mathrm{~m} / \mathrm{s}$ (easterly). Further analysis is discontinued until detailed corrections for individual thruster firings can be made. 


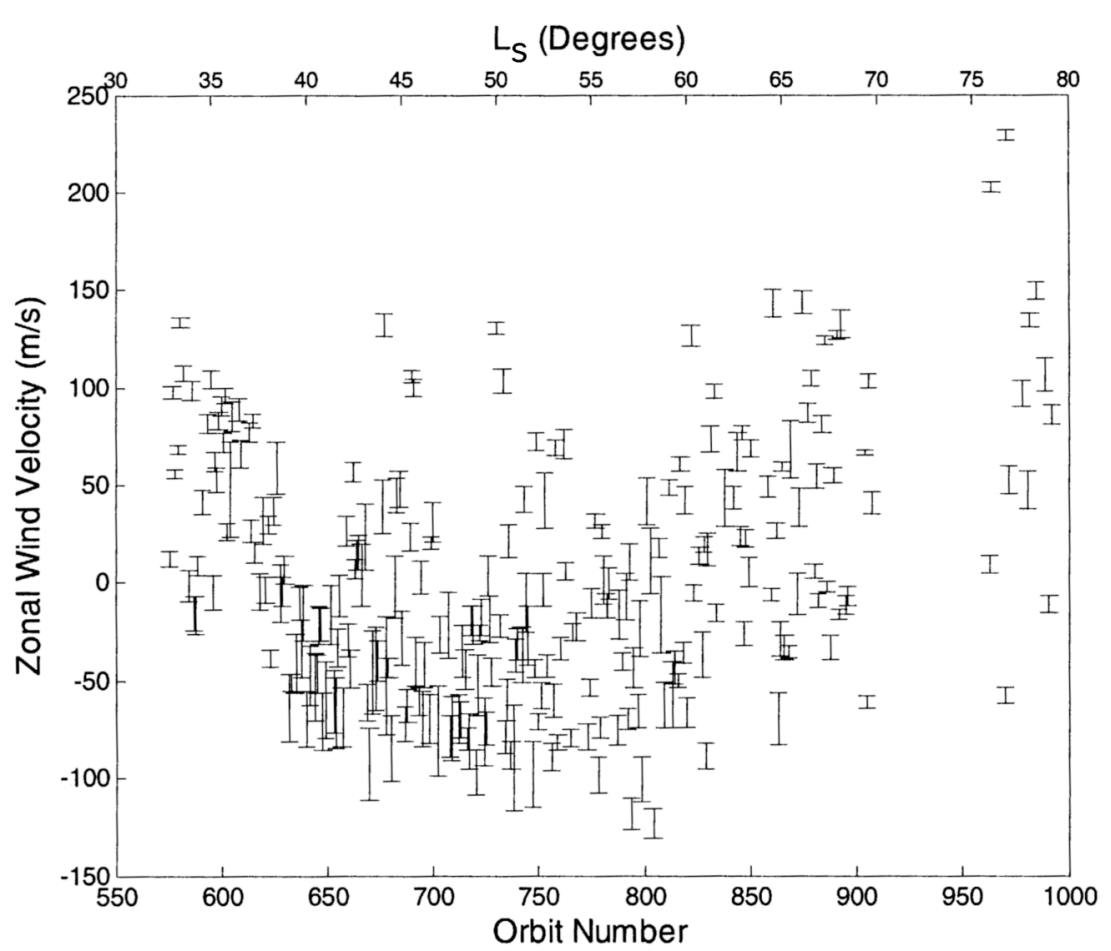

Figure 11. Computed phase 2 zonal winds with 3- $\sigma$ error bars.

\section{Seasonal Effects}

During phase 1, zonal winds were highest during southern spring (local northern fall) and tended to decrease as the southern summer season progressed. Phase 1 westerly zonal winds are consistent with northern hemisphere $\left(37^{\circ}-60^{\circ} \mathrm{N}\right)$ temperatures that are generally decreasing with increasing latitude. In addition, excessively high winds are most likely to occur when dust storms erupt, which is common in the southern spring season.

During phase 2 the zonal winds decrease during early northern spring $\left(60^{\circ}\right.$ to $0^{\circ} \mathrm{N}$ sampling) and then increase during late southern fall (southern hemisphere sampling). The latter illustrates westerly winds (up to $+150 \mathrm{~m} / \mathrm{s}$ ), again consistent with temperatures that generally decrease toward the local winter pole. ${ }^{9}$

\section{Conclusion}

The primary objective of Mars Global Surveyor aerobraking operations was to reduce the orbital period and eccentricity. However, data taken during aerobraking operations has proven useful in determining zonal wind patterns. The results of the research documented in this report show that winds during phase 1 of aerobraking are primarily westerly. However, on occasion, wind patterns were shown to be easterly. The easterly winds occurred only at latitudes between $\sim 45^{\circ}$ and $60^{\circ}$. During a regional dust storm, which began at about orbit 49 and lasted for a number of weeks, extremely high westerly winds (up to $300 \mathrm{~m} / \mathrm{s}$ ) occurred suddenly, rising more than $100 \mathrm{~m} / \mathrm{s}$ above the "normal" wind velocity.

Discontinuities in the zonal wind model were reduced by determining the average panel bias at the periapsis of each orbit and adjusting the spacecraft center of mass to account for the center of mass offset that results from such a deflection.

Noticeable and significant seasonal effects are observed. The latitude of wind sampling must be considered when describing seasonal effects. For example, westerly zonal winds are strongest during northern hemisphere $\left(37^{\circ}-60^{\circ} \mathrm{N}\right)$ in late fall when temperatures are generally decreasing with increasing latitude. Southern hemisphere westerly zonal winds are strongest during local southern winter, when temperatures are generally decreasing toward the local winter pole. Furthermore, excessively high winds are most likely to occur when dust storms erupt, a common occurrence in the southern spring season. 


\section{Appendix}

Flow Chart to Solve for Zonal Winds

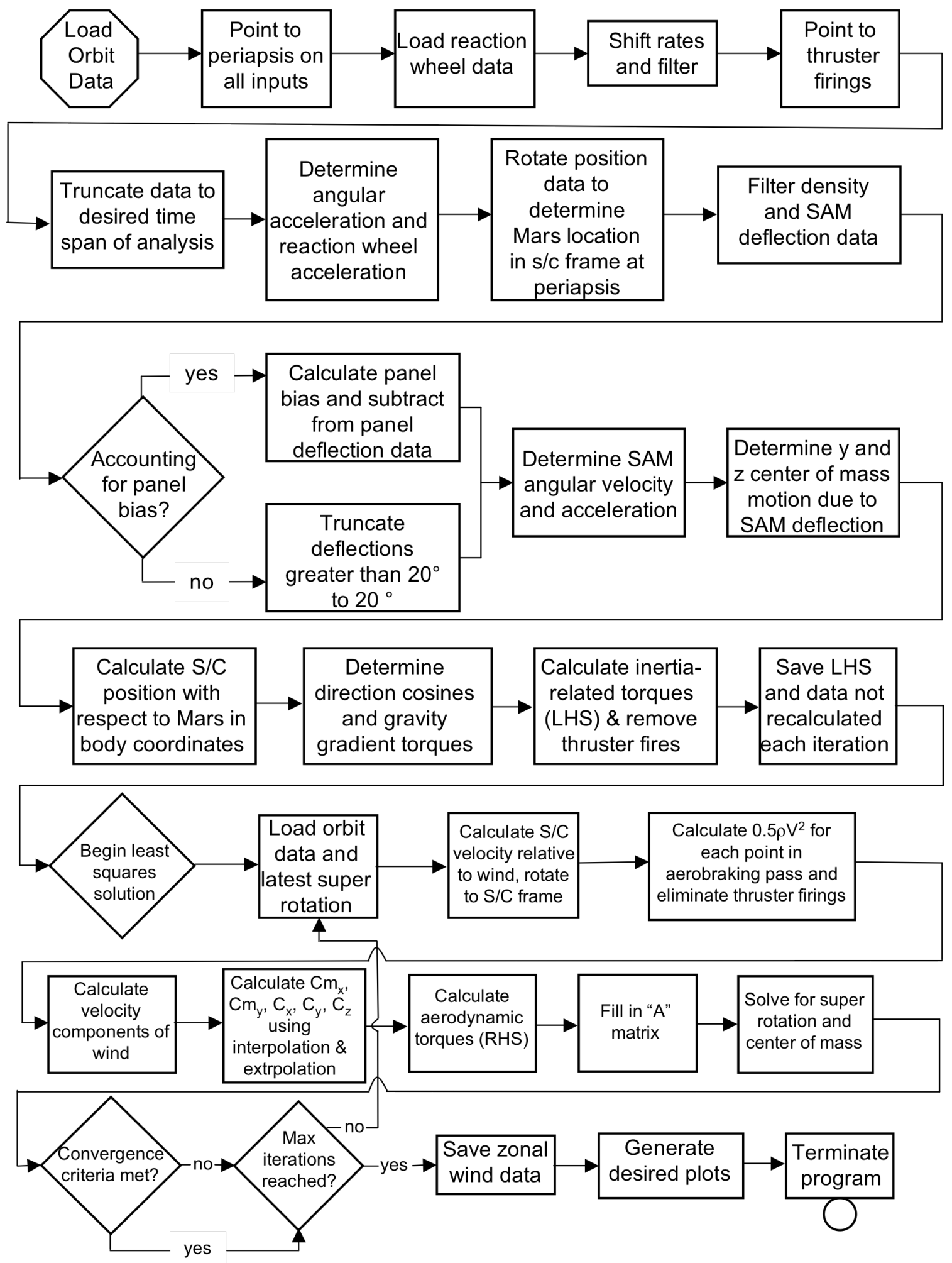




\section{Acknowledgments}

The work described in this paper was started at the George Washington University, Joint Institute for Advancement of Flight Sciences, sponsored by the National Aeronautics and Space Administration, and was completed at the Jet Propulsion Laboratory, California Institute of Technology, under a contract with the National Aeronautics and Space Administration. NASA Mars Data Analysis Program support for S. Bougher is also recognized.

\section{References}

${ }^{1}$ Dallas, S. Sam, "Mars Global Surveyor Mission.” IEEE Aerospace Conference, Vol. 4, IEEE, 1997, Piscataway, NJ, pp. 173-189.

${ }^{2}$ Tolson, R.H. and Keating, G.M., et al, "Application of Accelerometer Data to Mars Global Surveyor Aerobraking Operations," Journal of Spacecraft and Rockets, Vol 36, No. 3, May-June, 1999, pp. 323-329.

${ }^{3}$ Keating, G.M., et al., "The Structure of the Upper Atmosphere of Mars: In-Situ Accelerometer Measurements from the Mars Global Surveyor," Science, vol. 279, March 13, 1998, pp. 1672-1676.

${ }^{4}$ Cancro, G.J., R.H. Tolson, and G.M. Keating, "Operational Data Reduction Procedure for Determining Density and Vertical Structure of the Martian Upper Atmosphere from Mars Global Surveyor Accelerometer Measurements," NASA/CR1998-208721, 1998, pp. 37-38.

${ }^{5}$ Meirovitch, L., Methods of Analytical Dynamics, McGraw-Hill, New York, 1970, pp. 437.

${ }^{6}$ Wilmoth, R.G., et al., "Rarefied Aerothermodynamic Predictions for Mars Global Surveyor," Journal of Spacecraft and Rockets, Vol. 36, No. 3, May-June, 1999, pp. 314-322.

${ }^{7}$ Lyons, Daniel T., et al., "Mars Global Surveyor: Aerobraking Mission Overview," Journal of Spacecraft and Rockets, Vol. 36, No. 3, May-June, 1999, pp. 307-313.

${ }^{8}$ Justh, H. L., C. G. Justus, and V. W. Keller, "Global Reference Atmospheric Models, Including Thermospheres, for Mars, Venus, and Earth", AIAA/AAS Astrodynamics Specialist Conference, AIAA-2006-6394, AIAA, Wasington D.C., Aug. 2006 (to be published).

${ }^{9}$ Bougher, S. W., J. R. Murphy, J. M. Bell, M. A. Lopez-Valverde, and P. G. Withers, "Polar Warming in the Mars Lower Thermosphere: Seasonal Variations Owing to Changing Insolation and Dust Distributions," Geophysical Research Letters 33 (2): Art. No. L02203, Jan 272006. 